\title{
Pérdida de asignaturas en los estudiantes de enfermeria: aspectos económicos, familiares y psicosociales
}

\author{
Loss of subjects in nursing students: \\ economical, psychosocial and family related aspects
}

\author{
Diana C Tiga L ${ }^{1}$, Claudia E Cardozo $\mathrm{O}^{2}$, María T Fajardo $\mathrm{P}^{2}$, Diana C Vargas $\mathrm{H}^{4}$
}

Forma de citar: Tiga DC, Cardozo CE, Fajardo MT, Vargas DC. Pérdida de asignaturas en los estudiantes de enfermería: aspectos económicos, familiares y psicosociales. Rev Univ Ind Santander Salud. 2016; 45(4): 486-495. DOI: http://dx.doi. org/10.18273/revsal.v48n4-2016007 (c) (1)

\section{RESUMEN}

Introducción: El análisis de las asignaturas predice el desempeño académico y favorece la ejecución de intervenciones para el mejoramiento estudiantil. Objetivo: Evaluar la pérdida de asignaturas y la existencia de factores asociados; tales como los sociodemográficos, académicos, familiares, económicos y de salud, en estudiantes de enfermería de la Universidad Industrial de Santander durante el primer año de su carrera. Métodos: Estudio de corte transversal analítico, realizado en estudiantes durante su primer año de estudios, a partir de información académica y psicosocial, recogida entre el 2005 y 2012. Se realizó una regresión binomial negativa para identificar los factores relacionados al número de materias no aprobadas. Resultados: 171 (63.33\%) estudiantes no aprobaron alguna asignatura y en promedio un estudiante perdió 1,92 materias. La mayor pérdida se encontró en asignaturas de ciencias básicas. Como factores asociados a la disminución del número de materias reprobadas, se identificaron variables como trabajar antes de presentar el examen de estado para el ingreso a la educación superior, nivel educativo de la madre y depresión; en contraste, mayores ingresos, disfunción familiar, ansiedad y uso de cigarrillo, se asociaron a una mayor pérdida de materias. Conclusión: Las materias de ciencias básicas son factores académicos que influyen en la pérdida de materias de estudiantes de enfermería; igualmente, aspectos familiares como su funcionalidad, ingreso económico, educación de la madre y condiciones psicosociales como depresión, ansiedad y consumo de cigarrillo, pueden afectar el éxito o fracaso en las asignaturas.

Palabras Clave: Educación en enfermería. investigación en enfermería. relaciones familiares. depresión. ansiedad, hábito de fumar, rendimiento escolar bajo.

\begin{abstract}
Introduction: The analysis of subjects predicts academic performance and promotes the implementation of interventions for student improvement. Objective: To evaluate the loss of subjects and the existence of associated factors such as socio-demographic, academic, family, economic and health in nursing students from the Industrial

1. Instituto de Salud Pública de México

2. Universidad Industrial de Santander. Bucaramanga, Colombia

Correspondencia: María Teresa Fajardo Peña. Dirección: Carrera 32 N. 29-31. Correo electrónico: mariatefajardop@yahoo.es. Celular: +7 301 2384304
\end{abstract}


University of Santander during the first year of their career. Methods: Analytic cross-sectional study, perform in students during their first year of study, from academic and psychosocial information collected between 2005 and 2012. A negative binomial regression was performed to identify factors related to the number of subjects not passed. Results: $171(63.33 \%)$ students did not pass a subject and a student lost an average of 1.92 subjects. The greatest loss was found in basic science subjects. As associated factors with the decrease in the number of subjects failed, variables such as work before presenting the state exam for admission to higher education, educational level of the mother and depression were identified; in contrast, higher income, family dysfunction, anxiety and cigarette use were associated with a greater loss of subjects. Conclusion: The subjects of basic science are academic factors that influence the loss of subjects of nursing students; equally familiar aspects as functionality, income, maternal education and psychosocial conditions such as depression, anxiety and cigarette smoking, can affect the success or failure in the subjects.

Keywords: Education, nursing, nursing research, family relations, depression, anxiety, smoking, underachievement.

\section{INTRODUCCIÓN}

El bajo rendimiento académico del estudiante universitario ha sido motivo de interés y de múltiples investigaciones; pues es bien sabido, que la vida universitaria le impone una alta exigencia académica que requiere de un proceso de adaptación y asimilación, influenciado por una serie de factores que finalmente repercuten en su desempeño estudiantil $1^{1}$. El problema de bajo rendimiento se hace más evidente durante el primer año de estudios, produciendo altos índices de pérdida de asignaturas, que lleva a la repitencia y/o deserción, lo cual genera gran impacto en el ámbito social, económico y educativo ${ }^{2}$; lo anterior, es ratificado por el Ministerio de Educación Colombiano, al estudiar la deserción en la educación superior, donde encontró que ante un aumento de $1 \%$ en la tasa de repitencia, el riesgo de desertar en el siguiente semestre se incrementa en $1.47 \%$, siendo mayor en aquellos estudiantes con mal rendimiento académico ${ }^{3}$.

Es así, como el éxito estudiantil ha sido medido desde diferentes variables como es el promedio académico semestral o anual, el puntaje en una prueba determinada, la cantidad de exámenes adeudados, la nota media de estudios universitarios, la pérdida de materias, los años de carrera cursados, la percepción por parte del estudiante y/o la deserción, entre otros ${ }^{4-15}$.

Por su parte, en el reglamento institucional de la Universidad Industrial de Santander ${ }^{16}$, el rendimiento académico está medido por el promedio académico y el índice de aprobación; sin embargo, pese a lo mencionado existe escasa evidencia para analizar los factores asociados a la pérdida de asignaturas en un programa académico de pregrado y en particular en la carrera de enfermería ${ }^{9,17-20}$.

Por lo anterior, y teniendo en cuenta que el rendimiento académico merece ser una prioridad en la agenda de las 487 instituciones y de la política educativa ${ }^{2}$, en Colombia y en particular en la Universidad Industrial de Santander, se hace necesario realizar estudios que aborden dicha problemática y contribuyan a la identificación de factores influyentes en la pérdida de materias; es así, como este estudio pretende evaluar la pérdida de asignaturas y la existencia de factores asociados como económicos, familiares y psicosociales, en los estudiantes de enfermería de la Universidad Industrial de Santander (UIS) durante el primer año de su carrera.

\section{MATERIALES Y MÉTODOS}

Estudio de corte transversal de tipo analítico, realizado en estudiantes de enfermería de la Universidad Industrial de Santander, que cursaron al menos un núcleo temático (asignatura), durante su primer año de estudios entre el 2005 y 2012.

La información fue recolectada de tres fuentes: las variables académicas como promedio de calificaciones, asignaturas cursadas y sus respectivas notas; además de algunas variables sociodemográficas como ciudad y colegio de procedencia, fueron proporcionadas por la Dirección de Admisiones y Registro Académico de la universidad. La información relacionada con los aspectos psicosociales como APGAR familiar, Test de Zung para ansiedad y depresión, Test de CAGE para consumo de alcohol y encuesta VESPA para consumo de cigarrillo y sustancias psicoactivas, fue suministrada por la División de Bienestar Universitario de la universidad.

En cuanto a los instrumentos mencionados, el APGAR familiar es un método diseñado por el médico neonatólogo Gabriel Smilkstein en 1978, para detectar el grado de funcionalidad (o disfunción) de la familia. Consiste en cinco preguntas que mediante una escala de 0 a 4, evalúa el estado funcional de la familia. Sus parámetros se delinearon sobre la premisa que los 
miembros de la familia perciben el funcionamiento familiar y pueden manifestar el grado de satisfacción en el cumplimiento de los parámetros básicos de la función familiar en los atributos de: adaptación, participación, ganancia o crecimiento y afecto. Establece cuatro categorías: disfunción familiar severa $0-9$, moderada 10-13, leve 14-17, buena función familiar 18-20 $0^{21,22}$.

La escala de Zung para ansiedad, fue creada por $\mathrm{W}$ Zung para medir el nivel de ansiedad, esta cuenta con 20 preguntas; 15 de ellas relacionadas hacia los niveles de ansiedad mayores y cinco hacia la disminución. Cada pregunta se califica en una escala de 1-4 (ninguna o una pequeña parte del tiempo, parte del tiempo, buena parte del tiempo, la mayoría de veces) y sus puntajes son divididos en 4 categorías: normal sin psicopatología 20-39, ansiedad leve o ligera $40-47$, moderada 48-55 y severa o extrema $56-80^{23,24}$.

La escala de Zung para ansiedad, fue diseñada por W. Zung para cuantificar el nivel de ansiedad. El cuestionario contiene 20 preguntas y cEach question is scored on a scale of 1-4 (none or a little of the time, some of the time, good part of the time, most of the time). ada una se califica en una escala de 1-4 (ninguna o una pequeña parte del tiempo, parte del tiempo, buena parte del tiempo, la mayoría de las veces). There are fifteen questions worded toward increasing anxiety levels and five questions worded toward decreasing anxiety level Quince preguntas están redactadas hacia los niveles de ansiedad mayores y cinco hacia la disminución. The scores range from 20-80. Los puntajes pueden oscilar entre 20 y 80, divididos en cuatro categorías: dentro de lo normal sin psicopatología 20-39, ansiedad leve o ligera $40-47$, moderada $48-55$ y severa o extrema $56-80^{23,24}$.

El cuestionario CAGE, es un instrumento de tamizaje en el abuso de alcohol. Fue desarrollado por Ewing y Rouse en 1968 para detectar bebedores rutinarios. En 1974, Mayfield publicó el primer estudio de validación y posteriormente su fiabilidad y validez ha sido bien documentada en diferentes medios (hospital, otras áreas clínicas) y en el ámbito poblacional. Los primeros tres ítems del cuestionario CAGE son consistentes y complementarios con los criterios de abuso y dependencia del Diagnostic and Statistical Manual of Mental Disorders (DSM). Está constituida por cuatro preguntas calificadas como si o no, para un rango total entre 0 y 4 . Según el número de respuestas afirmativas, se considera que cuanto mayor es, mayor será también la dependencia. De este modo, se categoriza como no alcohólico entre $0 \mathrm{y}$, alto riesgo de alcoholismo 2 y alcohólico entre 3 y $4^{25}$.
El cuestionario VESPA: Sistema de Vigilancia Epidemiológica para Uso Indebido de Sustancias Psicoactivas, diseñado para unificar y consolidar la información epidemiológica sobre esta problemática. El sistema utiliza varias estrategias epidemiológicas como las encuestas de prevalencia y la vigilancia por unidades centinela. Estas últimas son centros públicos y privados especializados en la atención de farmacodependientes y en salud mental ${ }^{26}$.

Adicionalmente, la información se complementó con datos obtenidos del programa SPADIES versión 2.8. acerca de: composición familiar, principalmente nivel educativo de la madre; estatus laboral, referido a si el estudiante trabajaba o no antes de presentar el ICFES; y estatus económico, representado en ingresos en salarios mínimos.

Se protegió la privacidad de los datos, eliminando información que identificara al participante. Solo se empleó el código estudiantil. Los datos fueron manejados únicamente por los investigadores.

Se estableció como variable independiente el conteo de materias pérdidas durante el primer año de estudios universitarios y las variables sociodemográficas, académicas y psicosociales, como explicatorias. Para encontrar diferencias en el promedio de materias perdidas en las variables sociodemográficas, académicas y psicosociales, se realizó el test de ANOVA y para determinar los factores asociados a la pérdida de materias, se realizó un análisis múltiple mediante un modelo de regresión binomial negativa, teniendo como variable de respuesta el conteo de las materias perdidas. Inicialmente la información fue unificada en una base de datos en EXCEL, a partir del código del estudiante y analizada en el programa estadístico STATA SE 13.

\section{RESULTADOS}

Se recogieron datos de 270 estudiantes que ingresaron a partir de 2005, lo que corresponde al 53.25\% del total de estudiantes aceptados en el programa de enfermería; de éstos, 207 (76,6\%) fueron mujeres, 214 (79,29\%) provenían de colegio público y 200 (74,07\%) procedían del área metropolitana.

A continuación se presentan las variables que indican el nivel económico, la composición familiar y algunas características psicosociales de los estudiantes. Tabla 1. 
Salud Vol.48 No.4 Octubre - Diciembre de 2016

TABLA 1. Características familiares, económicas y psicosociales de los estudiantes de enfermería a su ingreso a la universidad.

\begin{tabular}{|c|c|c|c|c|}
\hline Variable & $\mathbf{N}$ & $\begin{array}{c}\text { Promedio } \\
\text { de materias } \\
\text { perdidas }\end{array}$ & ${ }^{\mathrm{a} D S}$ & Valor $\mathbf{p}$ \\
\hline \multicolumn{5}{|c|}{ Trabajaba al presentar el ICFES } \\
\hline No trabaja & 237 & 1.97 & 2.50 & $0.19 a$ \\
\hline Si trabaja & 9 & 0.88 & 0.60 & \\
\hline \multicolumn{5}{|l|}{ Salario Familiar } \\
\hline Menos de 1 & 18 & 2.22 & 3.04 & $0.80 ¥$ \\
\hline Entre 1 y 2 & 145 & 1.83 & 2.27 & \\
\hline Entre 2 y 3 & 49 & 2.02 & 2.63 & \\
\hline Entre 3 y 5 & 19 & 2.05 & 2.67 & \\
\hline Entre 5 y 10 & 3 & 2.00 & 2.70 & \\
\hline Más de 10 & 11 & 2.27 & 3.19 & \\
\hline Sin dato & 24 & 1.70 & 2.77 & \\
\hline \multicolumn{5}{|l|}{ Estrato } \\
\hline 1 & 35 & 1.68 & 2.09 & $0.69 ¥$ \\
\hline 2 & 98 & 1.87 & 2.16 & \\
\hline 3 & 100 & 2.09 & 2.94 & \\
\hline 4 & 36 & 1.83 & 2.40 & \\
\hline 5 & 1 & 0 & & \\
\hline \multicolumn{5}{|c|}{ Número de hermanos } \\
\hline 0 & 79 & 1.94 & 2.64 & $0.16 ¥$ \\
\hline 1 & 99 & 1.86 & 2.46 & \\
\hline 2 & 21 & 2.76 & 2.71 & \\
\hline 3 & 11 & 2.45 & 3.26 & \\
\hline Sin dato & 60 & 1.56 & 2.06 & \\
\hline \multicolumn{5}{|l|}{ Año de ingreso } \\
\hline 2005 & 39 & 2.38 & 2.97 & $0.27 * * *$ \\
\hline 2006 & 42 & 1.30 & 1.94 & \\
\hline 2007 & 37 & 3.10 & 3.47 & \\
\hline 2008 & 39 & 1.89 & 2.32 & \\
\hline 2009 & 25 & 2.12 & 2.61 & \\
\hline 2010 & 38 & 1.50 & 2.07 & \\
\hline 2011 & 48 & 1.37 & 1.57 & \\
\hline 2012 & 2 & 2.50 & 0.70 & \\
\hline \multicolumn{5}{|c|}{ Nivel educativo de la madre } \\
\hline Básica primaria & 47 & 2.51 & 2.93 & $0.10 ¥$ \\
\hline Secundaria & 126 & 1.84 & 2.27 & \\
\hline Media voc. Técnico & 47 & 1.44 & 2.09 & \\
\hline Universidad & 26 & 2.23 & 2.98 & \\
\hline Sin dato & 24 & 1.70 & 2.77 & \\
\hline
\end{tabular}

Variable

Promedio

$\mathbf{N}$ de materias aDS Valor $p$ perdidas

\begin{tabular}{lcccc}
\hline \multicolumn{4}{l}{ Test de Zung para ansiedad } \\
\hline Normal & 234 & 1.96 & 2.53 & $0.36 ¥$ \\
Ansiedad leve & 33 & 1.48 & 2.19 & \\
Ansiedad moderada & 3 & 3.33 & 2.30 &
\end{tabular}

Test de Zung para depresión

\begin{tabular}{lcccc}
\hline Normal & 254 & 1.98 & 2.53 & $0.26 ¥$ \\
Depresión leve & 13 & 1.00 & 1.63 & \\
Depresión Grave & 3 & 0.66 & 1.15 & \\
\hline
\end{tabular}

Apgar familiar

\begin{tabular}{lccccc}
\hline $\begin{array}{l}\text { Buena } \\
\text { familiar }\end{array}$ & función & 140 & 1.64 & 2.24 & $0.19 ¥$ \\
$\begin{array}{l}\text { Disfunción } \\
\text { leve }\end{array}$ & familiar & 81 & 2.07 & 2.58 & \\
$\begin{array}{l}\text { Disfunción familiar } \\
\text { moderada }\end{array}$ & 34 & 2.29 & 3.07 & \\
$\begin{array}{l}\text { Disfunción familiar } \\
\text { severa }\end{array}$ & 15 & 2.80 & 2.62 & \\
\hline
\end{tabular}

\section{Test CAGE}

\begin{tabular}{lllll}
\hline Sin alcoholismo & 222 & 1.78 & 2.39 & $0.67 ¥$
\end{tabular}

$\begin{array}{lllll}\text { Alto } & \text { riesgo } & 10 & 2.40 & 2.63\end{array}$

$\begin{array}{llll}\text { alcoholismo } & 10 & 2.40 & 2.63 \\ \text { Alcoholismo } & 2 & 2.50 & 0.71\end{array}$

\begin{tabular}{lcccc} 
Alcoholismo & 2 & 2.50 & 0.71 & \\
\hline Uso de drogas & & & & \\
\hline Nunca & 265 & 1.91 & 2.50 & 0.39 a \\
Alguna vez & 5 & 2.20 & 1.92 & \\
Uso de alcohol & & & & \\
No usa & 173 & 1.87 & 2.56 & $0.61 ¥$ \\
Últimos 30 días & 53 & 2.07 & 2.37 & \\
Último año & 60 & 1.93 & 2.25 & \\
\hline Uso de cigarrillo & & & & \\
\hline No usa & 218 & 1.62 & 2.24 & $<0.01 ¥$ \\
Últimos 30 días & 30 & 3.43 & 2.66 & \\
Último año & 22 & 2.77 & 3.59 &
\end{tabular}

aDS: Desviación estándar, ${ }^{\mathrm{b}}$ a Ttest, $^{\mathrm{c}} ¥$ ANOVA. $* * *$ test de tendencia entre grupos ordenados

En relación con la pérdida de materias, $171(63.33 \%)$ estudiantes reprobaron alguna asignatura durante su primer año de estudios y en promedio, 1 estudiante perdió 1,92 asignaturas. Los años en los que hubo mayor pérdida de materias fueron 2005, 2007 y 2009.

Se encontró una diferencia estadísticamente significativa en el promedio de materias perdidas 
Pérdida de asignaturas en los estudiantes de enfermería: aspectos económicos, familiares y psicosociales

en cuanto al uso del cigarrillo, observando que los estudiantes que han fumado en los últimos 30 días, perdieron 3,43 materias; mientras que aquellos que no fuman, solo perdieron en promedio 1.62 materias (ANOVA, valor $\mathrm{p}<0.01$ ). Tabla 1.

$\mathrm{Al}$ observar la dinámica de pérdida de materias durante el periodo de estudio, se encontró que las materias que con mayor frecuencia reprobaron los estudiantes fueron: en el primer semestre, biociencias 1 y para el segundo semestre, biociencias 2 y morfofisiología general; estas asignaturas, corresponden al componente de ciencias básicas del currículo. Figuras 1 y 2.

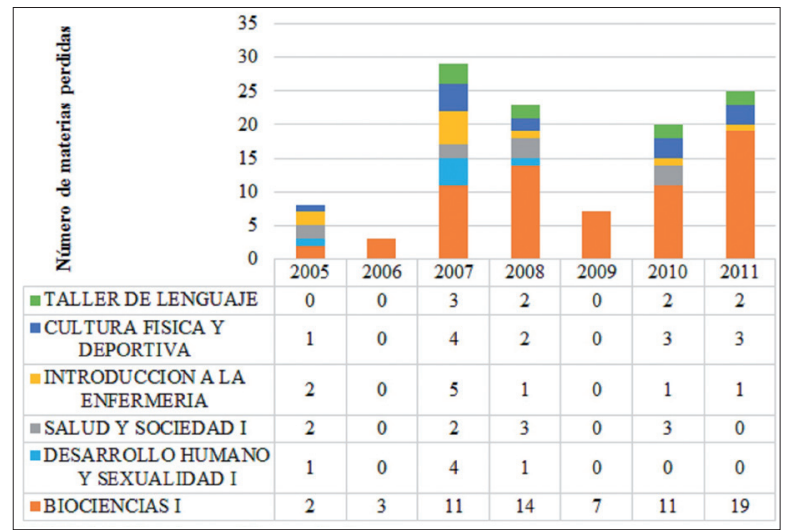

FIGURA 1. Materias perdidas en el primer semestre

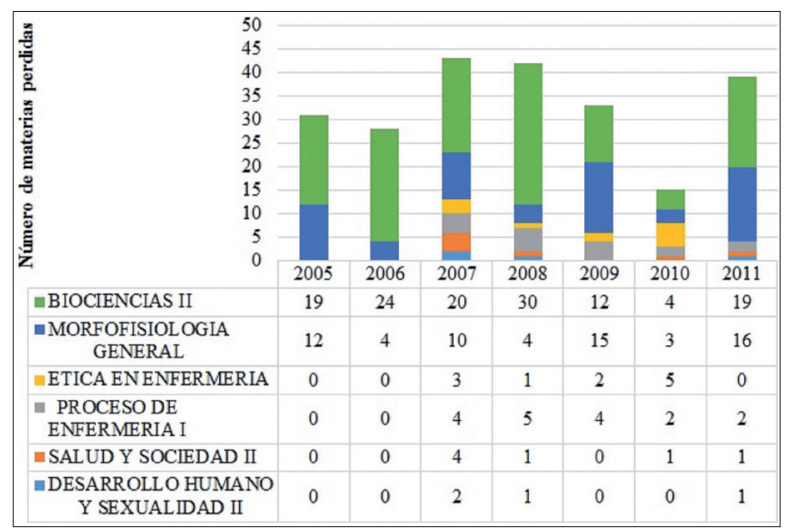

FIGURA 2. Materias perdidas en el segundo semestre

Al realizar el análisis múltiple mediante regresión binomial negativa, para evaluar los factores asociados al número de materias perdidas, se encontró que: haber trabajado antes de presentar el examen para el ingreso a la educación superior - ICFES, el nivel educativo de la madre y la depresión del estudiante, se asocian a una reducción en el número de pérdida de materias en el primer año; de manera contraria, tener mayores ingresos familiares, la disfunción familiar, la ansiedad y el uso de cigarrillo, se asociaron a un aumento en la pérdida de materias. Tabla 2.
TABLA 2. Modelo de regresión binomial negativa para conteo de materias perdidas en el primer año.

\begin{tabular}{|c|c|c|c|c|c|}
\hline \multirow{2}{*}{$\begin{array}{l}\text { Variables } \\
\text { Sexo }\end{array}$} & \multirow[t]{2}{*}{${ }^{a}$ IRR } & \multirow[t]{2}{*}{$\begin{array}{c}\text { berror } \\
\text { Est. }\end{array}$} & \multirow[t]{2}{*}{$\begin{array}{c}\text { Valor } \\
\mathbf{p}\end{array}$} & \multicolumn{2}{|c|}{${ }^{\mathrm{c}} \mathrm{IC} 95 \%$} \\
\hline & & & & & \\
\hline Hombre & Ref & - & - & - & - \\
\hline Mujer & 0.81 & 0.15 & 0.24 & 0.57 & 1.15 \\
\hline \multicolumn{6}{|c|}{ Trabaja al presentar el ICFES } \\
\hline No & Ref & - & - & - & - \\
\hline $\mathrm{Si}$ & 0.29 & 0.09 & $<0.01$ & 0.16 & 0.53 \\
\hline \multicolumn{6}{|l|}{ Salario } \\
\hline Menos de 1 & 0.82 & 0.28 & 0.57 & 0.42 & 1.61 \\
\hline Entre 1 y 2 & Ref & - & - & - & - \\
\hline Entre 2 y 5 & 2.00 & 0.44 & $<0.01$ & 1.29 & 3.10 \\
\hline 5 o más & 1.70 & 0.83 & 0.28 & 0.64 & 4.44 \\
\hline \multicolumn{6}{|l|}{ Educación de la madre } \\
\hline Básica primaria & Ref & - & - & - & - \\
\hline Secundaria & 0.58 & 0.13 & 0.01 & 0.37 & 0.89 \\
\hline Media voc. Técnico & 0.29 & 0.10 & $<0.01$ & 0.16 & 0.56 \\
\hline Universidad & 0.44 & 0.21 & 0.09 & 0.19 & 1.13 \\
\hline \multicolumn{6}{|l|}{ APGAR } \\
\hline Buena función familiar & Ref & - & - & - & - \\
\hline $\begin{array}{l}\text { Disfunción familiar } \\
\text { leve }\end{array}$ & 1.29 & 0.21 & 0.13 & 0.93 & 1.78 \\
\hline $\begin{array}{l}\text { Disfunción familiar } \\
\text { moderada }\end{array}$ & 1.48 & 0.33 & 0.08 & 0.96 & 2.28 \\
\hline $\begin{array}{l}\text { Disfunción familiar } \\
\text { severa }\end{array}$ & 2.73 & 0.92 & $<0.01$ & 1.43 & 5.20 \\
\hline \multicolumn{6}{|l|}{ Depresión Zung } \\
\hline Normal & Ref & - & - & - & - \\
\hline Depresión leve & 0.38 & 0.12 & $<0.01$ & 0.20 & 0.70 \\
\hline Depresión Grave & 0.14 & 0.07 & $<0.01$ & 0.05 & 0.39 \\
\hline \multicolumn{6}{|l|}{ Ansiedad Zung } \\
\hline Normal & Ref & - & - & - & - \\
\hline Ansiedad leve & 0.67 & 0.18 & 0.13 & 0.40 & 1.12 \\
\hline Ansiedad moderada & 2.64 & 1.01 & 0.01 & 1.24 & 5.62 \\
\hline \multicolumn{6}{|l|}{ Uso del Cigarrillo } \\
\hline No usa & Ref & - & - & - & - \\
\hline Últimos 30 días & 1.49 & 0.29 & 0.04 & 1.01 & 2.19 \\
\hline Último año & 1.49 & 0.41 & 0.14 & 0.87 & 2.54 \\
\hline
\end{tabular}

aIRR: Razón de Tasas de Incidencia bọ Error Est: Error Estándar, cIC: Intervalo de Confianza

Se evidencia que trabajar antes de presentar el ICFES, reduce en $71,1 \%$ el número de materias perdidas, con respecto a los que no trabajaron. En relación con la educación de la madre, aquellos estudiantes con madres que han alcanzado un nivel de media vocacional/ 
técnico, tienen una reducción de $71 \%$ en el número de materias perdidas respectivamente; en comparación con aquellos cuya madre tiene estudios de básica primaria. Adicionalmente, tener depresión leve disminuye $62 \%$ el número de materias reprobadas y tener depresión severa disminuye $86 \%$ el número de materias reprobadas con respecto a los que no la tienen.

En contraste, suponiendo que el ingreso familiar de un estudiante es de uno a dos salarios mínimos, si este mismo estudiante tuviera un ingreso familiar entre dos y cinco salarios mínimos y lo demás se mantuviera constante, se aumentaría al doble el número de materias reprobadas.

De igual manera, un estudiante que tiene una disfunción severa en la familia pierde 2,7 veces más materias con respecto a los que tienen una función familiar normal. De otra parte, los estudiantes que tienen ansiedad moderada reprueban 2,64 veces más con respecto a los que no la tienen y los que usaron cigarrillo en los últimos 30 días, pierden 50\% más asignaturas en relación a los que refieren no fumar. Tabla 2.

\section{DISCUSIÓN}

A partir del análisis de datos, se concluye que un porcentaje considerable de estudiantes de enfermería (63.33\%), pierden alguna asignatura durante el primer año de estudios y en promedio un estudiante reprueba 1,92 materias durante este periodo; situación que vale la pena analizar, teniendo en cuenta lo encontrado por Ibarra $\mathrm{M}$, et al., en su trabajo sobre rendimiento académico, donde por cada materia que consiga aprobar el alumno durante su primer año de estudio, se incrementa en 1,94 veces la probabilidad de obtener un buen rendimiento académico a lo largo de su carrera $(\mathrm{p}<0.01)^{18}$.

Por su parte, una investigación que buscaba evaluar el rendimiento académico de los alumnos de la licenciatura en enfermería de la Universidad Nacional de Tucumán en Argentina, identificó que las materias biológicas presentan menor aprobación que las asignaturas profesionales ${ }^{27}$; caso similar al presente estudio, en donde el mayor número de asignaturas perdidas está en el área de ciencias básicas: biociencias I, biociencias II y Morfofisiología; las cuales son desarrolladas por docentes con formación en las áreas de biología, microbiología, medicina y química; quienes se encuentran adscritos al Departamento de Ciencias Básicas de la Escuela de Medicina de la universidad.

Así mismo, esta investigación evidenció que tener depresión leve o severa disminuye 62\% y $86 \%$ el número de asignaturas reprobadas con respecto a los 491 estudiantes que no tienen esta enfermedad; resultado opuesto a lo encontrado en la evidencia científica, en donde tener depresión es un factor asociado al rendimiento académico regular o malo; identificando además, que a mayores síntomas depresivos, menor es el rendimiento del estudiante. Uno de estos estudios encontró que $68 \%$ de los alumnos que tenían síntomas depresivos reprobaron alguna materia, lo que supuso un riesgo de reprobación de 2.4 veces, comparado con quienes no tenían dicha sintomatología ${ }^{33-36}$. Cabe mencionar que no se encontraron investigaciones que guardaran similitud con el presente estudio en lo que respecta a esta variable; sin embargo, en estudiantes de medicina una investigación mostró que la depresión tan solo afectó el promedio de dos asignaturas: anatomía $(\mathrm{p}=0.01)$ y salud pública $(\mathrm{p}=0.04)^{20}$.

En cuanto a los factores influyentes en la aprobación de asignaturas, se puede deducir que aquellos estudiantes que tienen la experiencia de la responsabilidad laboral en el momento de presentar el ICFES, muestran una mejor adaptación estudiantil y por consiguiente menor número de asignaturas perdidas; caso opuesto a lo encontrado en el análisis de deserción realizado por el Ministerio de Educación, en donde se evidencia que aquellos estudiantes que trabajan al presentar el ICFES, tienen un mayor riesgo de deserción ${ }^{3}$. Sin embargo, esta discrepancia no puede ser comparable, debido a que en los dos casos se evalúan los factores asociados a dos variables diferentes pérdida de asignaturas y deserción.

Otro factor protector que redujo la cantidad de pérdida de materias, es que la madre tenga un nivel educativo de secundaria o superior, resultado similar al estudio de tipo cuantitativo y de orden correlacional, realizado en la Universidad de Sucre ${ }^{5}$; a la revisión sistemática llevada a cabo por Garbanzo $\mathrm{G}^{30}$ y a la realizada por Montes IC, Lerner $\mathrm{J}^{31}$, en los cuales se evidencia que el nivel educativo de los progenitores, de la madre o del adulto responsable del estudiante, constituye un factor asociado al rendimiento académico. Por el contrario, un estudio transversal y correlacional realizado en cinco programas de psicología de la ciudad de San Juan de Pasto, encontró que tanto el nivel educativo de la madre como el del padre, no están correlacionados con el rendimiento académico del estudiante ${ }^{32}$.

Así mismo, esta investigación evidenció que tener depresión leve o severa disminuye $62 \%$ y $86 \%$ el número de asignaturas reprobadas; resultado opuesto a lo encontrado en la evidencia científica, en donde tener depresión es un factor asociado al rendimiento académico regular o malo; identificando además, que a mayores síntomas depresivos, menor es el rendimiento 
del estudiante. Uno de estos estudios encontró que $68 \%$ de los alumnos que tenían síntomas depresivos reprobaron alguna materia, lo que supuso un riesgo de reprobación de 2.4 veces, comparado con quienes no tenían dicha sintomatología ${ }^{33-36}$. Cabe mencionar que no se encontraron investigaciones que guardaran similitud con el presente estudio en lo que respecta a esta variable; sin embargo, en estudiantes de medicina una investigación mostró que la depresión tan solo afectó el promedio de dos asignaturas: anatomía $(\mathrm{p}=0.012)$ y salud pública $(\mathrm{p}=0.045)^{20}$.

Por otra parte, en este estudio se encontraron factores que aumentan la probabilidad de presentar un bajo rendimiento académico, como tener ingresos familiares altos; por lo que se podría pensar que tener bajos ingresos puede llevar al alumno a exigirse académicamente con el fin de no repetir asignaturas, evitar prolongar su carrera y por ende acarrear más gastos a su familia; resultado contrario a lo encontrado en estudiantes de la Universidad de Sucre, en donde aquellos estudiantes con ingresos familiares más altos, presentan condiciones más favorables para su rendimiento ${ }^{5}$. Una investigación realizada en la Escuela de Administración, Finanzas e Instituto Tecnológico de EAFIT, encontró que aquellos estudiantes con mejor nivel socioeconómico presentaron menor pérdida de materias ${ }^{31}$. Sin embargo, otras investigaciones identificaron que los aspectos socioeconómicos no influyen en el desempeño académico de los estudiantes ${ }^{20}$.

En relación con el componente familiar, presentar disfunción severa en las relaciones familiares aumenta el riesgo de reprobar una asignatura; caso similar a lo evidenciado por Labarca y Fuhrmann en su estudio descriptivo correlacional en estudiantes de medicina, el cual muestra una relación significativa entre las variables familiares y el rendimiento académico de los jóvenes, tendiendo a mejorar cuando existe una mayor cercanía con la madre ${ }^{11}$. Otro estudio realizado con estudiantes de Medicina de una universidad en México, encontró que los estudiantes con menor rendimiento académico presentaban mayor disfuncionalidad familiar ${ }^{37}$.

La ansiedad es otra variable que ha tenido impacto en el rendimiento académico del estudiante universitario, $\mathrm{y}$ en el presente estudio no es la excepción; encontrando que aquellos estudiantes que la presentan, reprueban mayor número de materias. Así mismo, una investigación realizada en Hong Kong, en enfermería, determinó que los estudiantes con niveles más altos de ansiedad, presentan más errores en su rendimiento clínico ${ }^{28}$. Otro estudio realizado en estudiantes de ingeniería identificó una correlación significativa entre altos niveles de ansiedad y bajo rendimiento académico ${ }^{38}$; opuesto a lo anterior, Alkhateeb H, Nasser R y Kholer, encontraron que la ansiedad es un predictor del éxito académico y además está relacionado con la motivación; es decir, la voluntad de invertir tiempo y esfuerzo para completar las tareas académicas ${ }^{8,39}$.

Finalmente, la literatura coincide con el resultado obtenido en la variable consumo de cigarrillo, en donde se establece una correlación estadísticamente significativa con el rendimiento académico regular o malo, encontrando que conforme se incrementa la prevalencia tanto en experimentación como en consumo actual de tabaco, decrece el desempeño estudiantil ${ }^{40,41}$. El consumo de alcohol y drogas no mostraron ningún impacto en el rendimiento académico; caso contrario a lo encontrado en otros estudios, en donde estas variables han sido predictoras de fracaso ${ }^{14,42}$.

Teniendo en cuenta que adicionalmente a los hallazgos, se encontró que durante los años 2007 y 2009 hubo mayor pérdida de materias, coincidiendo con reformas curriculares al plan de estudios en 2006 y 2009; en las cuales, se integraron algunas asignaturas y se modificaron requisitos y prerrequisitos; se considera que lo anterior pudo haber contribuido a que el rendimiento académico disminuyera; haciéndose necesario un mayor estudio y profundización de este aspecto en futuras investigaciones, a fin de identificar cómo las reformas curriculares pueden influir en el rendimiento académico.

\section{CONCLUSIONES}

En este estudio se encontró que trabajar antes de presentar el examen para el ingreso a la educación superior ICFES, el nivel educativo de la madre y la depresión del estudiante, se asocian a una disminución en el número materias perdidas en el primer año; de manera contraria, la disfunción familiar, la ansiedad y el uso de cigarrillo, se asociaron a un aumento en la pérdida me materias.

Teniendo en cuenta que las asignaturas con mayor pérdida son desarrolladas por docentes de áreas diferentes a enfermería, es importante un trabajo interdisciplinario que le brinde al estudiante con riesgo de bajo rendimiento académico, orientación, acompañamiento y asesoría, a través de herramientas para el mejoramiento y el fortalecimiento del desempeño universitario. 
De otra parte, la variable depresión al constituir un factor protector en el rendimiento académico, merece ser objeto de mayor estudio, a fin de dar explicación al papel que desempeña y determinar si su comportamiento está influenciado por el tipo de población. Para futuras investigaciones, se sugiere incluir factores como empleo alterno a los estudios, rendimiento en la secundaria, puntaje de admisión a la universidad, asistencia a clases, manejo del tiempo, uso de métodos de estudio, pedagogía y asesoría brindada por el docente; los cuales no fueron analizados en este estudio por diversas razones, pero que según la evidencia científica han demostrado tener asociación con el rendimiento académico, de manera que se implementen mejores

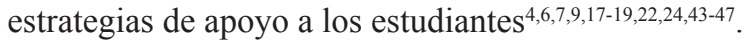

Se espera que con los resultados obtenidos, la Escuela de Enfermería lleve a cabo anualmente la evaluación de asignaturas perdidas y sus factores asociados, con el fin de establecer estrategias que permita intervenirlos; y de esta forma, generar impacto a corto y mediano plazo en el rendimiento académico de los estudiantes.

Considerando que son pocos los estudios sobre pérdida de asignaturas y sus factores asociados; este trabajo genera resultados valiosos, como referente nacional e internacional, que pueden ser tenidos en cuenta al investigar dicha problemática en una población similar; así mismo, constituye un punto de partida para la construcción de ambientes favorecedores del mejoramiento estudiantil desde diversos ámbitos: psicológico, académico, familiar, social y económico.

Para el grupo investigador es muy importante el estudio realizado dado que se adquiere el compromiso permanente de mejora al interior del currículo y, por ende, el fortalecimiento del programa de enfermería, con un mayor posicionamiento dentro de la institución educativa y de ésta en el país.

\section{AGRADECIMIENTOS}

Especiales agradecimiento a la Vicerrectoría de Investigación y Extensión de la Universidad Industrial de Santander por la financiación del proyecto de investigación. A la División de Bienestar Universitario y a la Dirección de Admisiones de la Universidad Industrial de Santander, por los datos suministrados en la etapa de recolección de la información.

\section{CONSIDERACIONES ÉTICAS}

El presente estudio se rige por los lineamientos de la Resolución 8430 de 1993 del Ministerio de Salud y se considera sin riesgo; además, fue aprobado por el comité de ética de la Universidad Industrial de Santander. La información se recolectó directamente de la historia clínica de ingreso de cada estudiante y nunca se empleó el nombre, solo el código; cumpliendo la normatividad de buenas prácticas. Los instrumentos utilizados no tuvieron ningún ajuste.

\section{CONFLICTO DE INTERESES}

No existió conflicto de interés para la realización del presente trabajo.

\section{REFERENCIAS}

1. Kaufman, RA. Planificación de los sistemas educativos: Ideas básicas concretas. $2^{\mathrm{a}}$ ed. México: trillas; 1998.

2. Ministerio de Educación Nacional. Diagnóstico de la deserción en Colombia. Educación Superior. Boletín informativo 14. 2010: 4-18.

3. Ministerio de Educación Nacional. Deserción Estudiantil en la Educación Superior Colombiana. Elementos para su diagnóstico y tratamiento. 2008: 1-132.

4. Lancia L, Petrucci C, Giorgi F, Dante A, Grazia Cifone M. Academic success or failure in nursing students: results of a retrospective observational study. Nurse Educ Today. 2013; 33(12): 1501-1505. DOI: $10.1016 /$ j.nedt.2013.05.001.

5. Mercano Covo TM, Niño Sotomayor CG. Factores académicos y personales asociados al rendimiento académico de los estudiantes del programa de administración de empresas de la Universidad de Sucre. Zona Próx. 2012; 16: 54-67.

6. McCarey M, Barr T, Rattray J. Predictors of academic performance in a cohort of pre-registration nursing students. Nurse Educ Today. 2007; 27(4): 357-364. DOI: 10.1016/j.nedt.2006.05.017.

7. Salamonson Y, Everett B, Koch J, Andrew S, Davidson PM. The impact of term-time paid work on academic performance in nursing students: a longitudinal study. Int J Nurs Stud. 2011; 49 (5): 579-585. DOI: 10.1016/j.ijnurstu.2011.10.012.

8. Kohler J. Rendimiento académico, habilidades intelectuales y estrategias de aprendizaje en universitarios de Lima. Liber. 2013; 19(2): 277-288.

9. Montero Rojas E, Villalobos Palma J, Valverde Bermúdez A. Factores institucionales, pedagógicos, psicosociales y sociodemográficos asociados al rendimiento académico en la universidad de costa rica: un análisis multinivel. RELIEVE. 2007; 13(2): 215-234.

10. Ayyash Abdo H, Sánchez Ruiz MJ. Subjective wellbeing and its relationship with academic 
achievement and multilinguality among Lebanese university students. Int J Psychol. 2012; 47(3): 192202. DOI: $10.1080 / 00207594.2011 .614616$.

11. Labarca A, Fuhrmann I. Familia, salud mental y rendimiento en estudiantes universitarios. De Familias y Terapias. 2011; 20(30): 35-43.

12. Elias H, Ping W, Chong Abdullah M. Stress and academic achievement among undergraduate students in University Putra Malaysia. Procedia Soc Behav Sci. 2011; 29: 646-655. DOI: 10.1016/j. sbspro.2011.11.288.

13. Contreras K, Caballero C, Palacio J, PérezA. Factores asociados al fracaso académico en estudiantes universitarios de barranquilla (Colombia). Psicol. Caribe. 2008; (22): 110-135.

14. Aertgeerts B, Buntinx F. The relation between alcohol abuse or dependence and academic performance in first-year college students. J Adolesc Health. 2002; 31(3): 223-225. DOI: 10.1016/S1054$139 X(02) 00362-2$.

15. Blackman I, Hall M, Darmawan I. Undergraduate nurse variables that predict academic achievement and clinical competence in nursing. Int Ed J. 2007; 8(2): 222-236.

16. Universidad Industrial de Santander (UIS). Colombia. Reglamento académico-estudiantil de pregrado. Acuerdo $\mathrm{N}^{\circ} 72$ de 1982. Compilación de normas vigentes. 2012.

17. Whytea D, Madigana V, Drinkwaterc EJ. Predictors of academic performance of nursing and paramedic students in first year bioscience. Nurse Educ Today. 2010; 31 (8): 849-854. DOI :10.1016/j. nedt.2010.12.021

18. Ibarra M, Michalus J. Análisis del rendimiento académico mediante un modelo logit. Rev Ing Industrial. 2010; 9(2): 47-55.

19. Martí Ballester C. Análisis de los factores que influyen en el desempeño académico de los alumnos de contabilidad financiera a través de modelos de elección binaria. R. Bras. Gest. Neg. 2012; 14(45): 379-399.

20. Vargas I, Ramírez C, Cortés J, Farfán A, Heinze G. Factores asociados al rendimiento académico en alumnos de la Facultad de Medicina: estudio de seguimiento a un año. Salud Ment. 2011; 34(4): 301-308.

21. Paz S, Aymat A. Problemas en el desempeño escolar y su relación con el Funcionalismo familiar en alumnos de egb. Rev Fac Med. 2007; 8(1):27-32.

22. Arias CL, Herrera A. El Apgar familiar en el cuidado primario de la salud. Colomb Med. 1994; 25(1): 2628.

23. Zung WWK. Self-report depression scale. Arch Gen
Psychiatry. 1965; 12: 63-70.

24. Smilkstein G. Family APGAR analysed. Fam Med. 1993; 5(2): 293.

25. Ewing JA. Detecting alcoholism: The CAGE questionnaire. JAMA. 1984; 252: 1905-1907. PMID: 6471323

26. Torres Y, Muñoz AL, Ramírez H. Vigilancia epidemiológica sobre el uso indebido de sustancias psicoactivas "VESPA" Medellín, Antioquia 1992. Bol Epidemiol Antioq. 1994; 19: 180-193.

27. Torres S. Rendimiento académico de los alumnos de la licenciatura en enfermería de la Universidad Nacional de Tucumán. Visión de Enfermería Actualizada. 2011; 7(27): 12-21.

28. Pitt V, Powis D, Levett Jones T, Hunter S. Factors influencing nursing students' academic and clinical performance and attrition: an integrative literature review. Nurse Educ Today. 2012; 32(8): 903-913. DOI: $10.1016 /$ j.nedt.2012.04.011.

29. Houglum J, Aparasu R, Delfinis T. Predictors of academic success and failure in a pharmacy professional program. Am J Pharm Educ. 2005; 69(3): 283-289.

30. Garbanzo G. Factores asociados al rendimiento académico en estudiantes universitarios, una reflexión desde la calidad de la educación superior pública. Educación. 2007; 31(1): 43-63.

31. Montes IC, Lerner J. Rendimiento académico de los estudiantes de pregrado de la Universidad EAFIT. Perspectiva cuantitativa. Grupo de Estudios en economía y empresa. 2010-2011: 1 -139.

32. Guevara P, Jaramillo R, Tovar Paredes. Factores familiares y su relación con el rendimiento académico en estudiantes de psicología. Rev Virt Univ Cat Norte. 2013; (40): 122-140.

33. Franco C, Gutiérrez S, Perea E. Asociación entre depresión y bajo rendimiento académico en estudiantes universitarios. Psicogente. 2011; 14(25): 67-75.

34. Campo A, González S, Sánchez Z, Rodríguez D, Dallos C, Díaz L. Percepción de rendimiento académico síntomas depresivos en estudiantes de media vocacional de Bucaramanga, Colombia. Arch Pediatr Urug. 2005; 76(1): 21-26.

35. Gutierrez JA, Montoya LP, Toro BE, Briñon MA, Rosas E, Salazar LE. Depresión en estudiantes universitarios y su asociación con el estrés académico. CES Medicina. 2010; 24(1): 7-17.

36. Fouilloux C, Barragán Pérez V, Ortiz León S, Jaimes Medrano A, Urrutia Aguilar ME, Guevara Guzmán R. Síntomas depresivos y rendimiento escolar en estudiantes de Medicina. Salud Ment. 2013; 36(1): 59-65. 
37. Osornio L, García L, Méndez AR, Garcés LR. Depresión, dinámica familiar y rendimiento académico en estudiantes de medicina. Arch Med Familiar. 2009; 11(1): 11-15.

38. Prima V, Muhammad A, Ahmad O, Tutut H, Suriya $\mathrm{K}$. The relationship between study anxiety and academic performance among enginneering students. Procedia Soc Behav Sci. 2010; 8: 490497. DOI 10.1016/j.sbspro.2010.12.067.

39. Alkhateeb H, Nasser R. Assessment of learning and study strategies of university students in Qatar using an Arabic translation of the learning and study strategies inventory. Psychol Rep. 2014; 114(3): 947-965. DOI: 10.2466/11.03.PR0.114k26w3.

40. Gómez A, Gil Y, Aranzalez V. Consumo de cigarrillo, ingesta de alcohol y su asociación con la percepción de bajo rendimiento académico en estudiantes de ingeniería de una universidad pública. Duazary. 2011; 8(2): 169-174. DOI: 10.21676/2389783X.215

41. Arillo E, Fernández E, Hernández M, Tapia M, Cruz A, Lazcano E. Prevalencia de tabaquismo y bajo desempeño escolar, en estudiantes de 11 a 24 años de edad del estado de Morelos, México. Salud Pública Méx. 2002; 44(1): 54-66.

42. Ansari W, Stock C, Mills C. Is alcohol consumption associated with poor academic achievement in university students?. Int J Prev Med. 2013; 4(10): 1175-1188.

43. Rochforda C, Connollyb M, Drennanb J. Paid part-time employment and academic performance of undergraduate nursing students. Nurse Educ Today. 2009; 29(6): 601-606. DOI: 10.1016/j. nedt.2009.01.004.

44. Bordes V, Arredondo P, Robinson S, Rund J. A longitudinal analysis of latina/o students' academic persistence. J. Of Hispanic High. Education. 2011; 10(4): 358-368. DOI: $10.1177 / 1538192711423318$.

45. Balduf M. Underachievement among college students. J. Adv. Acad. 2009; 20(2): 274-294. DOI: 10.1177/1932202X0902000204.

46. Sansgiry S, Bhosle M, Sail K. Factors that affect academic performance among pharmacy students. Am J Pharm Educ. 2006; 70(5): 1-9.

47. Bertoli T, Barba M, Milán I. La permanencia de los estudiantes con riesgo académico en la universidad de ciencias médicas. Didact Educación. 2014; 5(2): 137-151. 